\title{
Retinol sérico en mujeres mexicanas urbanas durante el periodo perinatal
}

Esther Casanueva, Lic. en N utr., ${ }^{(1)}$ Roxana Valdés-Ramos, D ra. en C..1 ${ }^{(1)}$ Frania Pfeffer, D ra. en C..1 Arlene Ricalde-Moreno, Lic. en N utr., ${ }^{(2)}$ Elsy García-Villegas, Lic. en $\mathrm{N}$ utr., ${ }^{(2)} \mathrm{C}$ arlos M eza, Q .F.B. ${ }^{(3)}$

\begin{abstract}
Casanueva E, Valdés-Ramos R, Pfeffer F, Ricalde-Moreno A, García-Villegas E, Meza C. Retinol sérico en mujeres mexicanas urbanas durante el periodo perinatal. Salud Publica Mex 1999;41:317-321.
\end{abstract}

\begin{abstract}
Resumen
Objetivo. Establecer la prevalencia de deficiencia de vitamina A durante el embarazo y la lactancia en un grupo de mujeres mexicanas urbanas. Material y métodos Se invito a participar a un grupo de gestantes que acudían a control prenatal, que carecían de patología agregada y contaban con menos de 20 semanas de embarazo. Fueron evaluadas tres veces durante el embarazo y hasta la semana 24 del posparto. La determinación de vitamina A en suero se realizó por cromatografía de líquidos de alta presión. Se consideró como punto de corte para riesgo de deficiencia una concentracion de retinol < $1.05 \mu \mathrm{mol} / \mathrm{L}$. Resultados En las tres evaluaciones durante la gestación las concentraciones de vitamina A fueron de $2.34 \pm 0.70,2.41 \pm 1.03$ y $1.86 \pm 0.66 \mu \mathrm{mol} / \mathrm{L}$, respectivamente, lo cual muestra una disminución significativa. Sin embargo, sólo 1/30 se ubicó por debajo del punto de corte aceptado para considerar riesgo de deficiencia y ninguno para deficiencia. Durante el posparto las concentraciones se conservaron relativamente constantes, alrededor de $2.10 \mu \mathrm{mol} / \mathrm{L}$. La práctica de la lactancia no tuvo impacto sobre las concentraciones séricas de retinol, no aś la pérdida de peso. Conclusiones En la población estudiada no se documentaron casos de deficiencia de vitamina A durante el periodo perinatal. La pérdida de peso materna tiene influencia sobre las concentraciones de retinol en el posparto.
\end{abstract}

Palabras clave: vitamina A; embarazo; lactancia materna; nutrición; México

\author{
Casanueva E, Valdés-Ramos R, Pfeffer F, \\ Ricalde-Moreno A, García-Villegas E, Meza C. \\ Serum retinol in urban Mexican women \\ during the perinatal period. \\ Salud Publica Mex 1999;41:317-321.
}

\begin{abstract}
A bstract
Objective To establish the prevalence of vitamin A deficiency during pregnancy and lactation in a group of urban Mexican women. Material and methods.A gro up of pregnant women who attended a prenatal care program were invited to participate. Subjects had no added pathologies and were less than 20 weeks pregnant. Evaluations were performed 3 times during pregnancy until 24 weeks postpartum.Vitamin A levels were determined in serum by high performance liquid chromatography (HPLC). The cut-off point to consider retinol deficiency was $<1.05 \mu \mathrm{mol} / \mathrm{L}$. Results During the 3 gestational evaluations, vitamin $A$ levels were $2.34 \pm 0.70,2.41 \pm 1.03$ and $1.86 \pm 0.66 \mu \mathrm{mol} / \mathrm{L}$, respectively, which shows a significant decrease. H owever, only $1 / 30$ was situated below the accepted cut-off point to consider risk of deficiency, and none for deficiency. During post-partum , concentrations remained relatively constant, approximately $2.10 \mu \mathrm{mol} / \mathrm{L}$. Lactation had no impact on serum retinol levels, while weight loss did. Conclusions $N 0$ cases of vitamin A deficiency were found in the studied population during the perinatal period. Maternal weight loss affects post-partum retinol levels.
\end{abstract}

Key words: vitamin A; pregnancy; breast feeding; nutrition; Mexico

(1) Departamento de Investigación en N utrición, Instituto $\mathrm{N}$ acional de Perinatología (IN Per), México.

(2) Departamento de Salud, U niversidad Iberoamericana, México.

(3) Subdirección de Investigación en Salud Pública, IN Per, México.

Fecha de recibido: 5 de agosto de 1998 • Fecha de aprobado: 5 de marzo de 1999

Solicitud de sobretiros: Esther C asanueva. Instituto N acional de Perinatología. Montes U rales 800, 11000 México, D.F., México. Correo electrónico: casanuev@ servidor.unam.mx 
D e acuerdo con la Organización Mundial de la Salud (OMS), la deficiencia de vitamina A es un problema frecuente entre las mujeres y niños de Latinoamérica y el Caribe. ${ }^{1,2}$ En el caso de México, a pesar de que se carece de información epidemiológica sobre el estado de nutrición en vitamina A de la población en general, de acuerdo con la misma fuente la deficiencia de vitamina A representa un grave problema de salud pública. Por otra parte, se reconoce que dicha deficiencia incrementa la susceptibilidad a las infecciones tanto en las mujeres como en los niños y que, junto con la deficiencia de hierro, la carencia de vitamina A es uno de los principales contribuyentes al pobre desempeño materno durante el embarazo y la lactancia, así como responsable, en buena medida, del retardo en el crecimiento en los niños pequeños..$^{1,2}$

La prevalencia de la deficiencia de vitamina A puede evaluarse a partir de la concentración de retinol en plasma y, en el caso de las mujeres que amamantan, de su concentración en la leche, con la ventaja de que esta última representa una técnica no invasiva que además permite evaluar la capacidad de la mujer para producir leche con concentraciones adecuadas de retinol y, al mismo tiempo, medir la ingestión de vitamina $\mathrm{A}$ del lactante. ${ }^{3}$ De hecho, se ha sugerido utilizar la concentración de retinol en leche como un indicador para evaluar el impacto de diversas intervenciones encaminadas a eliminar su deficiencia. ${ }^{4-7}$ Sin embargo, ha sido escaso el interés por los cambios en las concentraciones plasmáticas de vitamina A durante el embarazo y el posparto en la mujer. ${ }^{8}$

Con base en lo anterior, se consideró pertinente evaluar la concentración plasmática de retinol a lo largo de la gestación y en el periodo posparto en un grupo de mujeres que habitan en el área metropolitana de la Ciudad de México y que acudieron a control prenatal durante su embarazo.

\section{Material y métodos}

\section{Sujetos}

Entre 1995 y 1996, se invitó a participar en el estudio a 47 mujeres que contaban con 16 o menos semanas de gestación, con fecha de última menstruación segura, que acudían a control prenatal al Instituto Nacional de Perinatología (INPer), que cursaban con embarazo único, sin patología agregada y estaban clínicamente sanas. Una vez que se les informó acerca de las características del estudio, 82\% aceptaron participar y firmaron una carta de consentimiento informado. El Comité de Investigación y Etica del INPer revisó y aprobó el protocolo.
Al inicio del estudio se interrogó a las mujeres sobre su peso pregestacional y se les tomó la talla con un estadímetro ad hoc, a fin de calcular su índice de masa corporal (IMC) pregestacional. Se evaluó a las mujeres en tres ocasiones, en las semanas 16, 24 y 30 de gestación y posteriormente cada cuatro semanas a partir de la cuarta semana posparto, hasta llegar a la semana 24. En cada evaluación se determinó el peso (kg) sin ropa y con una bata de peso conocido en una báscula calibrada (Torino, con capacidad de $120 \mathrm{~kg}$ ), utilizando las técnicas convencionales. ${ }^{9}$ Se tomó una muestra de sangre venosa de $5 \mathrm{ml}$ en ayuno.

A partir de las cuatro semanas posparto se interrogó a la mujer sobre la práctica de la lactancia que seguía; para los fines del estudio se consideró como lactancia exclusiva aquella en la cual el niño recibió exclusivamente leche humana y ningún otro alimento, líquido o suplemento durante las primeras 20 semanas.

\section{A nálisis bioquímico}

La muestra para la cuantificación de vitamina A en suero se tomó en un tubo sin anticoagulante y se determinó por cromatografía de líquidos de alta presión (HPLC Pelkin-Elmer, modelo 250), utilizando acetato de retinil como patrón interno para obtener la curva estándar, con un detector ultravioleta a $280 \mathrm{~nm}$ de acuerdo con la técnica que desarrolló Driskell. ${ }^{10}$ Todas las determinaciones se realizaron por duplicado, con un coeficiente de variación de $3.6 \%$.

Como punto de corte de normalidad de la concentración sérica de vitamina A se consideró una concentración $<1.05 \mu \mathrm{mol} / \mathrm{L}$ de retinol, de acuerdo con lo que han sugerido varios autores., ${ }^{4,11,12}$

Para los fines de análisis se utilizaron medidas de dispersión y tendencia central. La comparación entre los valores gestacionales y posgestacionales se realizó por medio de ANOVA para una vía y se calculó la ecuación de regresión que mejor se ajustó a la distribución de los mismos. Los cambios en las concentraciones de vitamina A se analizaron en función de la ganancia/pérdida de peso durante el periodo de estudio y la práctica o no de la lactancia. Para establecer las diferencias de acuerdo con los cambios de peso corporal, los puntos de corte se definieron de acuerdo con la mediana de la distribución de la ganancia gestacional (peso alcanzado en la semana 36 de gestación menos peso pregestacional) o la pérdida de peso posparto (peso alcanzado en la semana 24 posparto menos peso en la semana 36 de gestación). Las diferencias entre los grupos se analizaron por medio de la prueba de $t$ para muestras independientes, con una $p<0.05$. 
La información se analizó en una computadora personal mediante el paquete estadístico para las ciencias sociales para Windows (SPSS).

\section{Resultados}

Del total de mujeres invitadas a participar, 30 cumplieron con todas sus evaluaciones y no se identificaron fuentes de sesgo respecto a las mujeres que desertaron, es decir, no existieron diferencias en cuanto a edad, escolaridad o concentraciones iniciales de retinol entre los grupos.

Como se puede observar en el cuadro I, se trata de un grupo de mujeres cuya edad promedio fue de 30.7 años. Solamente una de cada cuatro mujeres era primigesta. Todas recibieron complemento de sulfato ferroso durante la gestación y ningún suplemento vitamínico. El índice de masa corporal pregestacional (IMC) promedio se encontraba por arriba del límite para considerar sobrepeso (IMC $>25$ ); sin embargo, las mujeres presentaron una ganancia de peso adecuada $\mathrm{y}$, en promedio, tuvieron hijos de buen peso al nacer. Por lo que se refiere al posparto, la mitad de las mujeres alimentaron a sus hijos al pecho en forma exclusiva cuando menos hasta las 20 semanas de vida posnatal. En diez casos ofrecieron lactancia mixta o lactancia materna parcial a partir de la primera semana de vida y cinco de las mujeres no amamantaron. En cuanto a la escolaridad ésta fue de 11 años en promedio y al menos $50 \%$ completó el ciclo de la educación media.

En el cuadro II se presentan las concentraciones de vitamina A a lo largo del embarazo y el posparto. Como se puede observar, en la medida en que avanzó el embarazo las concentraciones de retinol disminuyeron significativamente (ANOVA $4.10 p=0.02$ ). En la

\section{Cuadro I \\ Características generales de mujeres urbanas EMBARAZADAS O EN LACTANCIA QUE PARTICIPARON EN EL ESTUdio. MÉXICO, 1996-1997}

\begin{tabular}{lcc} 
Características & Promedio $\pm \mathrm{DE}$ & Intervalo \\
Edad (años) & $30.7 \pm 6.0$ & $19-42$ \\
\hline Gestaciones & $3 \pm 2$ & $1-7$ \\
\hline IMC pregestacional & $23.8 \pm 3.1$ & $19.6-31.9$ \\
\hline Talla (cm) & $154.1 \pm 4.0$ & $145.7-160.8$ \\
\hline Ganancia de peso gestacional $(\mathrm{kg})$ & $10.1 \pm 3.8$ & $6.00-16.00$ \\
\hline Peso del recién nacido $(\mathrm{g})$ & $3159 \pm 555$ & $2050-4250$ \\
\hline Pérdida de peso posparto $(\mathrm{kg})$ & $7.03 \pm 3.7$ & $-2.00-14.00$ \\
$\mathrm{n}=30$ & & \\
IMC: índice de masa corporal & &
\end{tabular}

salud pública de méxico / vol.41, no.4, julio-agosto de 1999

\section{Cuadro II \\ Concentración de Vitamina A ( $\mu$ mol/L) \\ DE MUJERES EMBARAZADAS O EN LACTANCIA, SEgún PERIOdo de ESTUdio. México, 1996-1997}

Semanas Promedio $\pm D E \quad$ Intervalo \% de deficiencia*

Embarazo

\begin{tabular}{cccc}
16 & $2.34 \pm 0.70$ & $1.26-3.91$ & 0 \\
\hline 24 & $2.41 \pm 1.03$ & $1.11-6.42$ & 0 \\
\hline 36 & $1.86 \pm 0.66$ & $0.77-3.21$ & 10.0
\end{tabular}

Posparto

\begin{tabular}{llll}
4 & $2.17 \pm 0.50$ & $1.15-3.21$ & 0 \\
\hline 8 & $2.06 \pm 0.52$ & $1.15-3.66$ & 0 \\
\hline 12 & $2.13 \pm 0.55$ & $1.19-3.35$ & 3.3 \\
\hline 16 & $2.05 \pm 0.54$ & $0.87-3.46$ & 3.3 \\
\hline 20 & $2.13 \pm 0.66$ & $0.97-3.56$ & 3.3 \\
\hline 24 & $2.15 \pm 0.69$ & $0.94-3.77$ & 3.3
\end{tabular}

$*<1.05 \mu \mathrm{mol} / \mathrm{L}$

AN OVA de una vía

para los valo res gestacionales $F=4.10, p=0.02$

para los valores posparto $F=0.31, p=0.73$

$n=30$

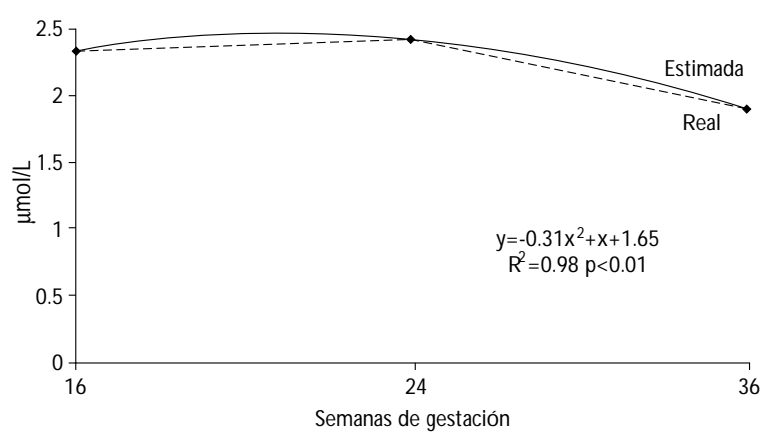

Figura 1. Retinol sérico según semana de embarazo

figura 1 se presenta la ecuación de regresión de los cambios gestacionales de vitamina $\mathrm{A}$, donde se puede observar una disminución de la semana 24 a la 36. Por su parte, el estado de nutrición a lo largo del periodo posgestacional tendió a conservarse constante y no fue posible identificar una disminución significativa en las concentraciones de vitamina $\mathrm{A}$ a lo largo de dicho periodo (ANOVA $0.17 p>0.05$ ). Cabe mencionar que únicamente se identificaron casos por debajo del punto de corte en la semana 34 de gestación y en las semanas 16, 20 y 24 posparto. En esos periodos se trató 
únicamente de un caso y no necesariamente fue la misma mujer en todas las evaluaciones. En ningún caso se identificaron signos clínicos de deficiencia.

Por otra parte, al analizar la evolución de la concentración de retinol a lo largo de la gestación fue imposible identificar las variables que la determinaron. Ni la paridad, ni el IMC pregestacional o la ganancia de peso permitieron discriminar entre diferentes patrones de comportamiento de la vitamina. Por lo que se refiere al patrón posparto se encontró que, de manera contraria a lo que podía esperarse, la práctica de la lactancia no tuvo influencia sobre la evolución del estado de nutrición en vitamina A (figura 2). Sin em-

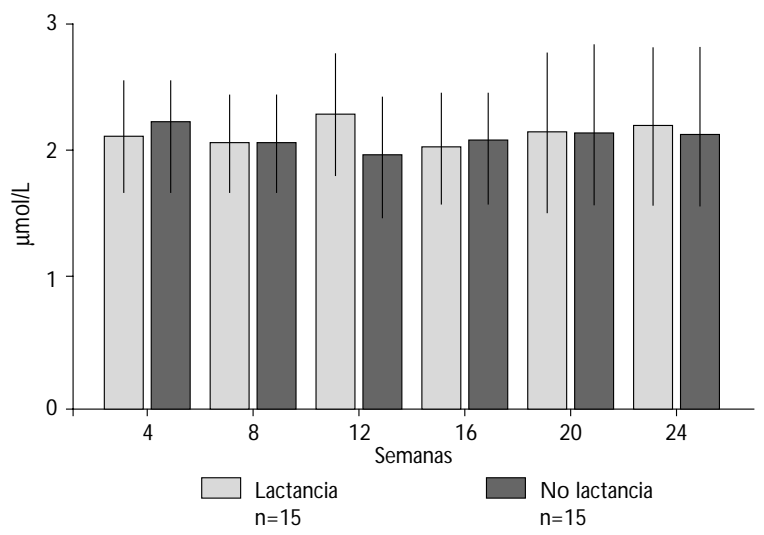

Figura 2. Concentración materna de vitamina A

\section{Cuadro III \\ Concentración de vitamina A $\mu$ mol/L EN EL POSPARTO, SEGÚN PÉRDIDA DE PESO CORPORAL. MÉxıco, 1996-1997}

\begin{tabular}{ccccc} 
Semanas & \multicolumn{2}{c}{ Pérdida de peso $\mathrm{kg}^{*}$} & & \\
\cline { 2 - 5 } & $<7.00$ (13 casos) & $\geq 7.00$ (17 casos) & Prueba de $t$ & $p$ \\
4 & $2.31 \pm 0.54$ & $2.06 \pm 0.45$ & 1.39 & 0.17 \\
\hline 8 & $2.09 \pm 0.55$ & $2.04 \pm 0.52$ & 0.25 & 0.81 \\
\hline 12 & $2.30 \pm 0.43$ & $1.99 \pm 0.61$ & 1.51 & 0.14 \\
\hline 16 & $2.30 \pm 0.58$ & $1.85 \pm 0.43$ & 2.49 & 0.02 \\
\hline 20 & $2.20 \pm 0.71$ & $2.07 \pm 0.64$ & 0.51 & 0.61 \\
\hline 24 & $2.48 \pm 0.78$ & $1.89 \pm 0.51$ & 2.51 & 0.02
\end{tabular}

* Respecto al peso en la semana 36 de gestación bargo, en el cuadro III se puede observar que las mujeres que a las 24 semanas posparto habían perdido al menos $7 \mathrm{~kg}$ respecto al peso alcanzado en la semana 36 de la gestación, tuvieron concentraciones significativamente más bajas de retinol en las semanas 16 y 24 que aquellas mujeres cuya pérdida de peso fue menor a $\operatorname{los} 7 \mathrm{~kg}$.

\section{Discusión}

Antes de iniciar con la discusión de los resultados sobre el estado de nutrición en vitamina A de las mujeres evaluadas, conviene hacer algunas reflexiones sobre las características de la muestra estudiada. Se trata de un grupo de mujeres urbanas que acuden a un servicio de salud, que se caracteriza por brindar atención prenatal a mujeres con embarazos de alto riesgo y sin seguridad social. Las mujeres que aceptaron participar en el estudio eran gestantes sin complicaciones, lo que las distingue del grupo de origen (gestantes con embarazos de alto riesgo). Adicionalmente se puede mencionar que, a juzgar por su escolaridad y su IMC pregestacional, no son diferentes del resto de las mujeres en edad reproductiva que habitan en el área metropolitana de la Ciudad de México ya que, de acuerdo con la Encuesta Nacional de Salud Materno Infantil realizada por la Secretaría de Salud en $1994,{ }^{13} 68 \%$ de las mujeres en edad reproductiva que viven en el DF y área conurbada cuentan con 12 o más años de estudio (la escolaridad promedio del grupo de estudio correspondió a $11.53 \pm 3.79$ años). Por lo que se refiere a los indicadores antropómetricos, según los resultados de la Encuesta Nacional de Nutrición (área metropolitana $)^{14}$ el promedio de talla e IMC pregestacional de las mujeres en edad reproductiva, corresponde a $153.4 \pm 6.5 \mathrm{~cm}$ y $23.1 \pm 4.6$, respectivamente, mientras que en la muestra estudiada los valores informados son $154.1 \pm 4.0$ y $23.8 \pm 3.1$. Así, con las reservas del caso, se puede aceptar que la población estudiada es similar a la que habita en el Distrito Federal.

Los resultados indican que la población estudiada presenta un adecuado estado de nutrición en vitamina A. De hecho, en la semana 36 del embarazo, que es el punto con la concentración promedio más baja, la misma corresponde a $1.86 \pm 0.66 \mu \mathrm{mol} / \mathrm{L}$, similar a la informada por Basu en gestantes canadienses (2.3 $\mu \mathrm{mol} / \mathrm{L})^{15}$ o por Chen y colaboradores en embarazadas de Taiwan $(2.2 \mu \mathrm{mol} / \mathrm{L}){ }_{1}^{16} \mathrm{y}$ se encuentra por arriba del límite superior encontrado en Indonesia (1.54 $\mu \mathrm{mol} / \mathrm{L})^{5}$ y Nepal $^{17}$ en mujeres que amamantan. En 
consecuencia, se puede concluir que no existe deficiencia de vitamina A. Lo anterior contrasta notablemente con la apreciación de la OMS, según la cual la deficiencia de vitamina A afecta del 26 al 55\% de la población mexicana. ${ }^{18}$

Por lo que se refiere a los cambios en las concentraciones de vitamina A a lo largo de la gestación, los resultados informados son similares a los que Van den Berg encontró en 70 mujeres holandesas, las cuales mostraron una tendencia a la disminución en la medida en que avanzaba la gestación. ${ }^{19}$ La disminución en la concentración de retinol puede deberse a dos causas, principalmente: la primera obedece al paso de cantidades importantes de vitamina A hacia el producto, sobre todo durante el tercer trimestre de la gestación. ${ }^{20}$ La segunda se da como resultado del incremento en el volumen plasmático; de hecho Van den Berg, al corregir la concentración de retinol en función del volumen plasmático, encontró que ésta se mantiene relativamente constante a lo largo de la gestación. Luego entonces, el impacto de la gestación sobre la reserva materna de vitamina A pudiera ser menor al esperado en mujeres que inician la gestación con un adecuado estado de nutrición.

En relación con los cambios en la concentración de retinol en el posparto, llama la atención que las concentraciones séricas se mantengan relativamente constantes, tanto entre las mujeres que amamantan como en aquellas que no están en lactancia (figura 2), lo que hace suponer que en poblaciones con adecuado estado de nutrición en vitamina $\mathrm{A}$, la práctica de la lactancia no tiene un efecto deletéreo cuando menos a nivel de concentraciones plasmáticas. Resta por evaluar el impacto de la lactancia sobre las reservas hepáticas de retinol. Este efecto no ha sido demostrado por otros autores, ya que la evaluación del estado de vitamina A en la mujer se suele limitar a las gestantes y las que amamantan, pero sin estudiar a mujeres en el posparto que no amamantan. ${ }^{4,6,12}$

Un hallazgo interesante de este trabajo, es el hecho de que la pérdida de peso en el posparto, más que la práctica de la lactancia, estableciera diferencias en las concentraciones de retinol, sobre todo en las semanas 16 y 24 posparto. Lo anterior pudiera estar relacionado con las modificaciones dietéticas que realizan las mujeres para perder peso durante ese periodo; sin embargo, en este estudio se carece de elementos para probar dicha hipótesis.

\section{Referencias}

1.Underwood BA. HipovitaminosisA: epidemiología del problema de salud pública y estrategias para su prevención y control. Bol 0 ficina Sanit Panam 1994;117:496-505.

2.Underwood BA, Arthur P.The contribution of vitaminA to public health. FASEB J 1996; 10:1041-1048.

3. Underwood BA.The role of vitamin A in child growth, development and survival.Adv Exp Med Biol 1994;352:201-208.

4. 0 rtega RM, Andrés P, Martínez RM, López-Sobaler AM.Vitamin A status during the third trimester of pregnancy in Spanish women: Influence on concentrations of vitamin A in breast milk. Am J C lin N utr 1997;66: 564-568.

5. Stoltzfus RJ, Underwood BA. Breast-milk vitamin A as an indicator of the vitamin $A$ status of women and infants. Bull World Health O rgan 1995;73:703-711.

6.Tanumihardj SA, Muherdiyantiningsi $H$, Permaesih D. D aily supplements of vitamin $A(8.4 \mathrm{mmol} / \mathrm{L}, 8000 \mathrm{UI})$ improve the vitamin A status of lactating Indonesian woman. Am J Clin N utr 1996;63;32-35.

7. Suharno D, W est CE, Muhilal P, Karyadi D, Hautvast JGAJ. Supplementation with vitamin $A$ and iron for nutritional anaemia in pregnant women in W est Java, Indonesia. Lancet 1993;342:1325-1328.

8. Pee S, Yuniar Y, W est CE, Muhilal P. Evaluation of biochemical indicators of vitamin A status in breast-feeding and non-breast-feeding Indonesian women.Am J Clin N utr 1997;66:160-167.

9. Lohman TG, Roche AF, Martorell E. Anthropometric standardization reference manual. Illinois: Human Kinetics Books, 1988.

10. Driskell W J, N eese JW, Bryant CC, Basher MM. Measurement of vitamin $A$ and vitamin $E$ in human serum by high-performance liquid chromatography. J Chromatography 1982;231:439-444.

11.Van den Berg H.Vitamin A intake and status. Eur J C lin N utr 1996;50 suppl 3:S7-S12.

12. Ackurt F, W etherilt $H$, Löker M, Hacibekiroglu M. Biochemical assessment of nutritional status in pre- and post-natal Turkish women and outcome of pregnancy. Eur J Clin N utr 1995; 49:613-622.

13. Dirección General de Atención Materno Infantil. Encuesta $N$ acional de Salud Materno Infantil 1994. México, D.F.: Secretaria de Salud, 1995:28. 14. González-C ossío T, Sanín LH, Hernández-Avila M, Rivera J, Hu H. Longitud y peso al nacer: el papel de la nutrición materna. Salud Publica Mex 1998:40:119-126.

15. Basu TK, W ein EE, Gango padhyay KC, W olever TMS, G odel JC. Plasma vitamin $A$ (retinol) and retinol-binding protein in newborns and their mothers. N utr Res 1994;14:9:1297-1303.

16. Chen HW, Lii CK, O u CC, W ong YC, Kuo BJ, Liu CH . Plasma vitamin A and $E$ and red blood cell fatty acid profile in newborns and their mothers. Eur J C lin N utr 1996;50:556-559.

17. Christian P, Schulze K, Stoltzfus RJ,W est KP Jr. Hyporetinolemia, illness symptoms, and acute phase protein response in pregnant women with and without night blindness. Am J C lin N utr 1998;67:1237-1243.

18. Ramakrishnan $U$, Martorell R. The role of vitamin A in reducing child mortality. Salud Publica Mex 1998;40:189-198.

19.Van den Berg H. Vitamin and mineral status in healthy pregnant women. En:Berger $\mathrm{H}$, ed.Vitamins and minerals in pregnancy and lactation. N estlé N utrition W orkshop Series. Raven: Raven Press, 1988: 93-108.

20. Underwood BA. Maternal vitaminA status and its importance in infancy and early childhood. Am J Clin N utr 1994;59 suppl:517-524. 